\title{
A study on prevalence of oral morbidities in an urban slum of Kishanganj district, Bihar, India.
}

\author{
${ }^{1}$ Dr. Nilanjana Ghosh, ${ }^{2}$ Dr. Kasif Shahnawaz, ${ }^{3}$ Dr. Soumya Kanti Bose, \\ ${ }^{4}$ Dr. Indranil Chakrabarti, ${ }^{5}$ Dr. Rama Ram \\ ${ }^{1,2}$ Assistant Professor, Department of Community Medicine, M.G.M Medical College, Kishanganj \\ ${ }^{3}$ Senior Resident, Department of Dentistry, M.G.M Medical College, Kishanganj \\ ${ }^{4}$ Assistant Professor, Department of Pathology, North Bengal Medical College \\ ${ }^{5}$ Professor and Head, Department of Community Medicine, M.G.M Medical College, Kishanganj
}

\begin{abstract}
:
Background: Good oral health reflects the overall health status of an individual. Gingivitis and periodontal diseases are the leading causes of oral morbidities. Wide range of factors cause oral morbidities like adopted changes in dietary patterns, lifestyle factors, faulty oral hygiene practices, addiction, neglect and ignorance towards dental hygiene. Few inevitable factors like aging, socioeconomic status also affect oral morbidity. Objectives: To assess the prevalence of oral morbidities and determine the factors associated with it along with providing appropriate health education on related and relevant issues. Methods: 110 study subjects were selected by simple random sampling and they were interviewed using a predesigned pretested semi- structured questionnaire. A thorough clinical assessment was also done by an expert. Results: The study revealed that $70.9 \%, 30.9 \%$ and $36.4 \%$ of study subjects had dental caries, periodontitis and bleeding gums respectively. Further $55.6 \%$ were addicted to pan, tobacco or smokeless tobacco and $53.6 \%$ had habit of sweet/junk food intake and $64.5 \%$ had poor oral hygiene practices. Oral morbidities were found to be significantly associated with factors like addiction, poor oral hygiene, intake of sweet/junk foods and age. Conclusion - Oral morbidities in various forms are substantially present in the area. Addressing to the causal issues may lead to their decline. However, a larger study with more representative sample is needed.
\end{abstract}

Key Words: oral morbidities, addiction, poor oral hygienic practices.

\section{Introduction:}

It is well known that a good oral health reflects the overall health status of an individual. Unfortunately it has been ignored and neglected for long leading to rise in oral morbidities. ${ }^{1}$ These morbidities are not mostly life threatening but cause severe inconvenience and pain and are of serious public health importance.

Infection of oral cavity along with teeth and gingival are very common and they also affect the supporting structures of teeth. Gingivitis and periodontal disease is the leading cause of oral morbidities in 8 to $10 \%$ of adult population. Incidence increases with age to as high as $34.5 \%{ }^{2,3}$ Dental plaques, dental caries, oral ulcers and oral cancers are also on the rise in recent times. Periodontal disease commonly assessed by Community Periodontal Index by Loe and Silness are majorly classified as gingivitis or periodontitis which is defined as "a group of inflammatory diseases affecting the surrounding tissues that support and anchor the teeth in their sockets." ${ }^{4,5}$ Presence of bacterial plaque or calculus on supragingival or subgingival tooth surfaces cause them and they result in severe destruction of gums, alveolar bone and outer layer of tooth root if left untreated. ${ }^{1,3}$ Adults suffer from chronic periodontitis whereas aggressive periodontitis is more common in the younger age group. ${ }^{1,4}$ Many other diseases have also been accounted with oral morbidities like cardiovascular disease, stroke, diabetes, pre-term and low-birth-weight infants. ${ }^{5,6,7}$

There are wide ranges of factors causing oral morbidities. Adopted changes in dietary patterns like junk foods, sweets, lifestyle factors, faulty oral hygiene practices, addiction, neglect and ignorance towards dental hygiene are few of them. Moreover, few inevitable factors like aging, socioeconomic status also influence the outcome of oral health. Unfortunately, faulty dietary practices and lifestyle factors have a more pronounced effect on urban population. ${ }^{1,8}$

Thus oral self care, as a part of general care, is of paramount importance and plays a pivotal role in preventing oral morbidities. It comprises of change in attitude of people towards oral health, prevention, timely and appropriate diagnosis and professional care seeking as and when required. ${ }^{8}$ To improve oral health of population, WHO has set promotion of self-care as one of the goals for the year $2020^{9}$ so that it is practiced widespread.

Thus assessing the status of oral morbidities in a community with a deep insight into their causative factors may pave way for desirable health outcomes on a long run. However there is a scarcity of 
epidemiological data on representative urban population. Thus the present study was envisaged in an urban slum of Kishanganj district, Bihar to assess the prevalence of oral morbidities in the area, to find out the various factors associated with them and also for awareness generation and providing appropriate health education on related and relevant identified areas. This would add to the existing database apart from aiding the people of the area for better oral self care in future.

\section{Materials and Methods:}

A descriptive, cross-sectional study was conducted in an urban slum of Dilawarganj, the urban field practice area of M.G.M Medical College and L.S.K Hospital in Kishanganj, Bihar for a period of three months. Adult population pertaining to the study criteria were included in the study. Permanent residents of the area, willing to participate in the study, who were systemically healthy and had more than 15 teeth, were included in the study. Documented person with mental disorders, pregnant and lactating females, or those who had underwent oral prophylaxis in last six months were excluded from the study. Prevalence of dental caries was taken as $50 \%$ (WHO Document), to achieve maximum sample size and non-response rate as $10 \%$, the final sample size was calculated at 110 . The study subjects were selected by simple random sampling using lottery method.

A predesigned pretested semi structured validated questionnaire was used and interview method was used to assess the various aspects. Relevant record review was done as and where required. Clinical examination was done by a dental expert and illuminated light source, mouth mirror, CPI Probe and explorer was used for the purpose.

The study subjects, their families and the local people of eminence were sensitised about purpose and benefits of the study after obtaining the required institutional ethical clearance and permission from requisite higher authorities. Informed consent was taken from all the study subjects. Their clinical examination was conducted with help of a dental expert under proper lighting and they were seated upright during the examination. Demographic information was recorded for each subject prior to clinical examination. A thorough clinical assessment was performed according to criteria laid in WHO survey guidelines ${ }^{4}$.

Few operational definitions were used as follows:

- Oral morbidities: Dental caries, periodontitis, bleeding gums, gingivitis, ulcerated lesions, stains, calculus, and halitosis were all taken in purview. Disorders relating to buccal mucosa, palate and base of tongue were not included for operational feasibility.

- Literacy: Over the age of seven years if can read and write with proper understanding.

- Poor oral hygiene: Irregular and improper brushing habits techniques. Brushing with neem stick or toothbrush were both included as brushing.

- Sweet/junk food intake- Excessive intake (more than 5 times/per week in last two months).

\section{Result:}

A total of 110 subjects were selected pertaining to the study criteria. There were no non-responders. Thus the final study subjects were 110. The results were as follows.

The age range was $(58.2 \pm 5.48)$ years. $69.1 \%$ of the study subjects were males. All the study subjects had access to health facilities and none of the females were pregnant.55.5\% subjects were literate. The study revealed that $70.9 \%, 30.9 \%, 36.4 \%, 8.2 \%$ and a majority of $50 \%$ of the study subjects had dental caries, periodontitis, bleeding gums, ulcerated lesions and stains and calculus respectively. The responses were multiple in nature. (Table1)

Further exploring into the causes, it was found that among the study subjects, $55.6 \%$ were addicted to either pan, tobacco or smokeless tobacco and $53.6 \%$ had habit of sweet/junk food intake. Poor oral hygiene practices like irregular and improper brushing habits, techniques were noted in $64.5 \%$ cases.17.3\%cases had documented history of drug intake, like tetracycline or phenytoin within last three years. Record review revealed $45.4 \%$ study subjects to be affected with diabetes, $28.2 \%$ with atherosclerosis and $24.5 \%$ with both the conditions simultaneously.

Various factors were studied which could play a causal part in oral morbidities. Using a Chi-Square test, it was inferred that oral morbidities were significantly associated with addiction $(\mathrm{p}=0.00)$, poor oral hygiene $(p=0.013)$, intake of sweet/junk foods, $(p=0.00)$, and with age $(p=0.00)$. Elderly age group (those more than 60 years) reported more oral morbidities. However there was no significant association noted with the maximum literacy status of the study subjects $(\mathrm{p}=0.75)$. (Table 2$)$

Their knowledge regarding oral morbidities was assessed and $74.6 \%$ were not aware about the ill effects of oral morbidities or their prevention and treatment. However only $24.9 \%$ subjects practiced oral self care practices and only $19.3 \%$ took oral health problems as a cause of seeking professional care. 


\section{Discussion}

Though the findings of 110 selected study subjects is not comparable to studies on a larger scale, however the findings of various studies were similar to the present study.

A study by Chauhan D et al, revealed overall prevalence of gingival diseases was $22.9 \%$, whereas in Kerala another study found prevalence of gingival disease to be $15 \% .{ }^{10}$

Moreover, Greene in India reported overt evidence periodontal diseases to be $90 \%$ among 11to17-year old subject with low socioeconomic status being the main cause. ${ }^{11}$ Interestingly, high prevalence of gingivitis (84.37\%) was found by Dhar et al and another study in Maharashtra (81\%). ${ }^{5,6}$ Lack of appropriate knowledge and hygienic practices along with negligent attitude toward oral hygiene were attributed as the main cause similar to the present study. ${ }^{1}$ A study done by WHO in Pondicherry found percentage of dental caries to vary from $73.3 \%$ among (35-44) years to 55.6\% among (65-74)years. The high prevalence of caries in this rural region was attributed to poor oral hygiene and low awareness. ${ }^{12}$

Similar to the present study, another study also found prevalence of gingival diseases to be significantly associated with age. In striking contrast, another study concluded decrease in prevalence of gingivitis with the increasing age, due to increased knowledge of appropriate brushing techniques with age. ${ }^{1}$

Tobacco use was found to be a significant factor in causing poor oral hygiene similar to the present study. Many studies have implicated smokeless tobacco use as a risk factor for the development of oral mucosal problems and tumors. A study revealed that higher prevalence of leukoplakia, erythroplakia and abnormal soft tissue growth among smokeless tobacco user than the non tobacco users were an added epidemiological evidence of oral effects of smokeless tobacco use. ${ }^{13}$

A study found prevalence of smokeless tobacco use to be $52.7 \%$, a finding similar to the present study. Adults attending family practice clinics in Karachi, Pakistan and sports athletes in United States of America also have more than half the population indulging in tobacco use. It is however less in African countries.

In contrast to the present study, another study found poor literacy status to be significantly affecting the oral hygiene, may be due to lack of awareness and appropriate knowledge on oral self care.

\section{Conclusion:}

The study concluded that majority of people suffered from oral morbidities in form of dental caries, bleeding gums, stains and calculus. They constitute a substantial amount of oral burden in the study area as well. Moreover, majority of the population were found to be addicted and suffering from poor oral hygiene due to various factors. Addressing these issues in form of awareness generation is of utmost importance to ensure optimum utilization of the health services for effective health outcomes.

\section{Recommendation:}

Proper oral hygiene education and designing effective and appropriate community friendly strategies may lead to optimum utilization of the services and favourable health indicators. Behaviour and action oriented programs are critical and play a pivotal role in preventing oral morbidities at all stages. ${ }^{1}$ Addiction needs to be strongly discouraged through both legal approach and by awareness generation, interpersonal communication, counselling and motivation.IEC materials and health education tools on related and relevant issues also play a defining role. However a larger study with a more representative sample is needed.

\section{Limitation}

Though imperative, it was beyond the scope of the study to check for fluoride levels in water due to logistic and human resource constraints.

\section{References:}

[1]. Chauhan D, Chauhan T, Sachdev V, Kirtaniya BC. A study of oral hygiene status and prevalence of gingival diseases in 9 and 12year-old school children of a northern hilly state, India. Int J Health Allied Science 2012; 1:258-62.

[2]. Jain DC. Epidemiological study of dental caries. Indian Journal of Community Health 1986, p32-6.

[3]. Carranza FA. Classification of diseases of the periodontium. In: Carranza FA, Newman MG, editors. Clinical Periodontology. 10 $0^{\text {th }}$ ed.Philadelphia: W.B. Saunders Company; 2006. p. 58-81

[4]. World Health Organization. Oral health surveys: Basic methods. $4^{\text {th }}$ ed. Geneva: WHO; 1997. p 36-8.

[5]. Dhar V, Jain A, VanDyke TE, Kohli A. Prevalence of gingival diseases, malocclusion and fluorosis in school-going children of rural areas in Udaipur district. J Indian SocPedodPrev Dent 2007; 25:103-5.

[6]. Bhayya DP, Shyagali TR, Mallikarjun K. Study of oral hygiene status and prevalence of gingival diseases in 10-12 year school children in Maharashtra, India. J Int Oral Health 2010; 2:21-6.

[7]. Pandit K, Kannan AT, Sarana K, Aggarwal K. Periodontal disease and dental caries in primary school children in rural areas of Delhi. Indian J Paediatr 1986; 53:525-9.

[8]. Gautam D K, Vikas J, Amrinder T, Rambhika T, Bhanu K. Evaluating dental awareness and periodontal health status in different socioeconomic groups in the population of Sundernagar, Himachal Pradesh, India. J Int Soc Prevent Communit Dentistry 2012; 2:53-7.

[9]. Hobdell M, Petersen PE, Clarkson J, Johnson N. Global goals for oral health 2020. Int Dent J 2003; 53:285-8. 
A study on prevalence of oral morbidities in an urban slum of Kishanganj district, Bihar, India.

[10]. Jose A, Joseph MR. Prevalence of dental health problems among school going children in rural Kerala. J Indian Soc Pedod Prev Dent 2003; 21:147-51.

[11]. Greene JC. Periodontal disease in India: Report of an epidemiological study. J Dent Res 1960; 39:302-12.

[12]. Ramachandran S, Singh Z, Senthivel. A study of dental morbidities among fishermen community in rural Tamil Nadu. Journal of Health and Community Dentistry 2013;7(1):8-11

[13]. Aqbor MA, Azodo CC, Tefouet TS. Smokeless tobacco use, tooth loss and oral health issues among adults in Cameroon. African Health Sci. Sep 2013; 13(3): 785-90.

Tables:

Table 1: Table showing the prevalence of various oral morbidities $\quad(n=110)$

\begin{tabular}{|l|l|l|}
\hline Oral Morbidities* & Number $(\mathbf{N})$ & Percentage (\%) \\
\hline 1.Dental Caries & 78 & 70.9 \\
\hline 2.Periodontitis & 34 & 30.9 \\
\hline 3.Bleeding gums & 40 & 36.4 \\
\hline 4.Ulcerated lesions & 9 & 08.2 \\
\hline 6.Stains and Calculus & 55 & 50.0 \\
\hline
\end{tabular}

*Multiple responses

Table 2: Table showing association of oral morbidities with the various factors $(n=110)$

\begin{tabular}{|c|c|c|c|c|}
\hline Factors & Oral Morbidity Present & $\begin{array}{l}\text { Oral Morbidity } \\
\text { Absent }\end{array}$ & Chi square value & $\mathrm{P}$ value, significance \\
\hline Addiction & 52 & 9 & 13.64 & 0.00 , significant \\
\hline Poor oral hygiene & 56 & 15 & 06.16 & 0.00 ,significant \\
\hline $\begin{array}{l}\text { Sweet/junk food } \\
\text { intake }\end{array}$ & 49 & 10 & 17.43 & 0.00 ,significant \\
\hline 60 yrs. and above & 48 & 11 & 14.23 & 0.00 ,significant \\
\hline Literate & 44 & 17 & 0.10 & $\begin{array}{l}0.75, \\
\text { not significant }\end{array}$ \\
\hline
\end{tabular}

